\title{
STUDY ON DATA CLEANSING ALGORITHMS FOR OUTLIERS IN WATER SUPPLY SYSTEM
}

\author{
Jong Rib Kim, Gang Wook Shin, Sung Taek Hong and Dae Wook Kim \\ K-water, Daejeon, Korea
}

\begin{abstract}
There has been systematically sharing data between government agencies, local governments and public organizations and more public organizations are encouraged to open public data in Korea. In the water industry, research on artificial intelligence using big data, a technology related to the fourth industrial revolution, is currently being carried out actively. As a result, quality control of acquired data is necessary to secure the reliability of data by developing algorithms of data cleansing to minimize outliers. In this paper, LSTM (Long Short Term Memory) for cleansing outliers and missing data is proposed to improve data quality management.
\end{abstract}

\section{KEYWORDS}

Outlier, Data Cleansing, Water Supply, LSTM

\section{INTRODUCTION}

The waterworks are composed of various data tags. Water quality, flow rate, pressure and water level are very important data in the operation side of water purification plants, and numerous data to operate pumps, valves, etc. are organically linked (Jang, Lee, Hong, \& Chun, 2014). Unlike the relatively easy-to-detect missing data, it is difficult to detect inaccuracies in data. Therefore, various analysis studies have been conducted to clean abnormal data measured in the water treatment process such as the existing average imputation and the statistical method (correlation and regression) (Choi, Song, \& Kang, 2016; Kim, \& Lee, 2010). In research, quartiles and LSTM algorithms are studied to improve the reliability of cleaned data and try to implement them by open source.

\section{PROPOSED ALGORITHM}

The water industry is supposed to disclose major data to citizens and other relevant organization in Korea. Reliability is critical for these data, and the sensitive data to be corrected are selected in collaboration with manager and operational staff working in a plant. As shown in table 1,236 water data were selected for this research to develop the data cleansing to correct the outliers.

Table 1. List for Data Quality Management

\begin{tabular}{|c|c|}
\hline Item & Data List \\
\hline Public Open Data & Water Quality, Flow, Level, Pressure \\
\hline Data for Water Quality Management & Turbidity, Residual Chlorine, pH \\
\hline Data for Water Quantity Management & Flow, Level, Pressure, Chemical Dosage Rate \\
\hline Linked Data to Other Organization & Effluent Flow, Level, Pressure \\
\hline
\end{tabular}


At first, correlation analysis between the data is performed using the Minitab program as shown in Figure 1. 100,000 data were used for modelling which were collected one minute data during two month period and the five most correlated tags with an absolute value of 0.5 or more were selected. It has been found that the data tag "Transmission flow rate of the water pump in circulation" as shown in Table 2, has a high relationship with BR effluent pressure and HS input flow rate.

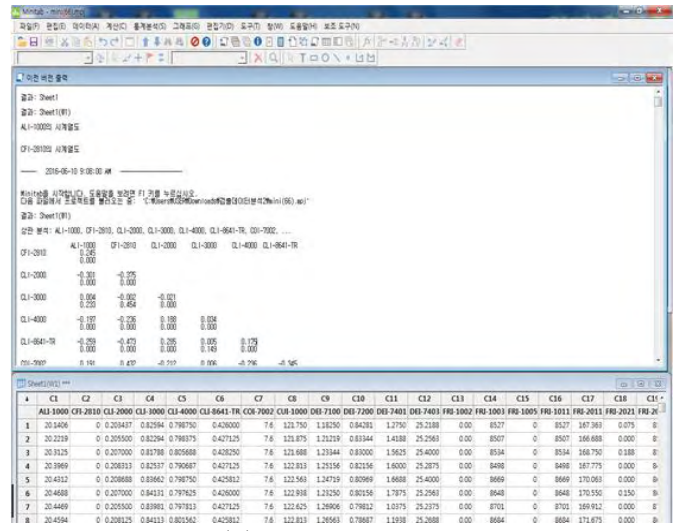

(a)

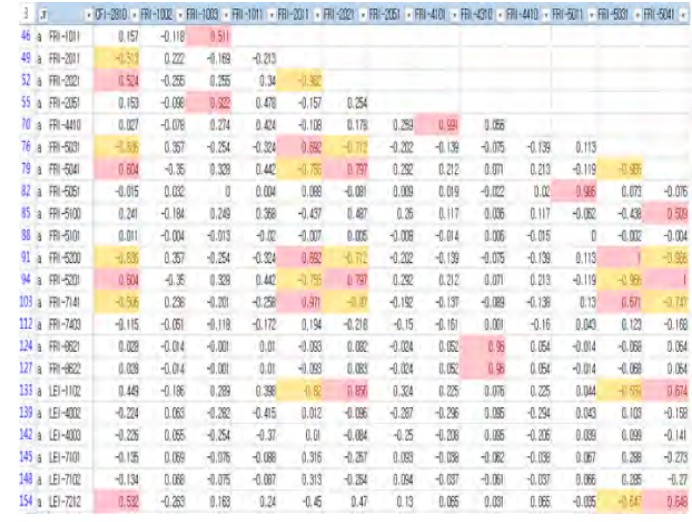

(b)

Figure 1. (a) Correlation Analysis by Minitap, (b) Correlation Analysis by Excel

Table 2. Correlation Result between Effluent Flow and Relevant Data

\begin{tabular}{|c|c|c|c|}
\hline Dependent Variable & No. & Independent Variable & Correlation Coefficient \\
\hline \multirow{5}{*}{$\begin{array}{c}\text { Effluent flow rate in BR } \\
\text { WTP }\end{array}$} & A & SC Pressure & 0.617 \\
\cline { 2 - 4 } & B & BR Effluent Pressure & 0.686 \\
\cline { 2 - 4 } & C & HS Pressure & -0.583 \\
\cline { 2 - 4 } & D & CD Pressure & -0.598 \\
\cline { 2 - 4 } & E & HS(P) Input Flow & 0.602 \\
\cline { 2 - 4 } & F & SS Input Flow & 0.551 \\
\cline { 2 - 4 } & H & HS Input Valve Position & 0.564 \\
\cline { 2 - 4 } & I & HS Input Flow & -0.623 \\
\cline { 2 - 4 } & J & WC Pressure & -0.62 \\
\hline
\end{tabular}

The equation using the regression algorithm is derived from the target data on the left and the independent variables on the right, as shown in Table 3. In the event of wrong and missing data, the correction value is derived by the corresponding equation.

Table 3. Cleansing Equation using Regression Method

$$
\begin{gathered}
\mathrm{Y}=0.617 * \mathrm{~A}+0.686 * \mathrm{~B}-0.583 * \mathrm{C}-0.598 * \mathrm{D}+0.602 * \mathrm{E}+0.551 * \mathrm{~F}+0.564 * \mathrm{G} \\
-0.623 * \mathrm{H}-0.62 * \mathrm{I}+0.525 * \mathrm{~J}
\end{gathered}
$$

Based on the correlation coefficient, the input variables are selected, and water quality data are corrected using the linear regression equation. It has an error of 2 to $30.9 \%$ depending on the input variables. Its disadvantage is that some highly correlated data can't be explained and water delay time is not considered. In addition, it is difficult to generalize a linear regression equation, which use different inputs to outlier and missing data. Thus, time series analysis needs to be considered to solve the delay time and generalize the algorithm.

We conduct outlier detection and imputation separately using artificial intelligence algorithm. First, the quantile method are proposed to detect the outlier as shown in Figure 2 (a), in which all of data is divided into five quantitation and the relevant data are grouped among them. One year data were used to make the quantile mapping. If it happen that new quantiled data don't belong to the existing mapping, LSTM proposes which 
value is replaced. Its structure is shown in Figure $2(\mathrm{~b})$. The algorithm use their own values as input $\{\mathrm{Y}(\mathrm{t}-1)$ to $\mathrm{Y}(\mathrm{t}-360)\}$ and output variables $\{\mathrm{Y}(\mathrm{t})\}$, which are applied to the all of data imputations to consider the time series characteristic. LSTM was compared with moving average method because both algorithms can work as autoregressive model.

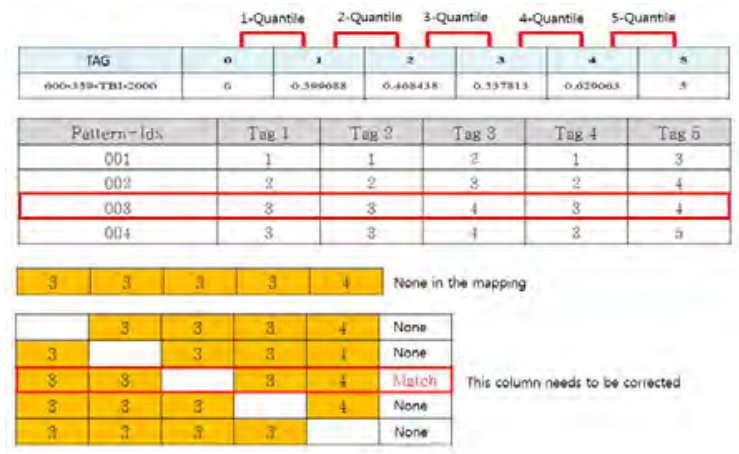

(a)

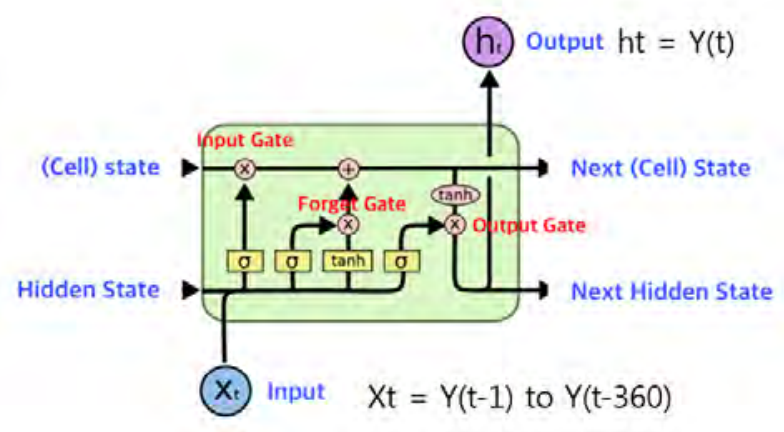

(b)

Figure 2. (a) Outlier Detection by Quantile Method, (b) Data imputation by LSTM

After the random outlier data from $20 \%$ to $100 \%$ of their true value is created for five target tags, the moving average (MA) and proposed methods were compared. Table 4 shows the difference of the existing Mean Imputation and LSTM method, in which it is shown that the error is improved by $5 \%$.

Table 4. Prediction Error between Mean and LSTM Imputation

\begin{tabular}{|c|c|c|c|c|c|c|} 
Imputation & Tag \#001 & Tag \#002 & Tag \#003 & Tag \#004 & Tag \#005 & Percentage Error \\
\hline MA & 5.376 & 20.147 & 22.433 & 6.923 & 16.624 & 14.3006 \\
\hline LSTM & 2.619 & 11.376 & 13.682 & 1.377 & 16.085 & 9.0278 \\
\hline
\end{tabular}

\section{CONCLUSION}

MA Imputation has the advantages of easy application and data correction by estimating missing values by the average of observed data values, but has the disadvantage of distorting statistical distributions in the wide range of missing data, which lead to the reduction of data reliability. LSTM is proposed to cope with those problem, which needs to be checked by the quantile method to detect abnormal data. Even though LSTM has a 5\% less error than MA, there is still a $9 \%$ error and it is thought to be that further research is needed.

\section{REFERENCES}

Choi, G.-W., Song, K.-S., \& Kang, J.. (2016). Understanding and policy assignment of R\&D of deep learning. KISTEP, Issue Paper 2016-08, 1-51, https://www.kistep.re.kr/c3/sub3.jsp?brdType=R\&bbIdx=10484

Jang, S.-B., Lee, H.-H., Hong, S.-T., \& Chun, M.G.. (2014). Development of Smart Remote Unit of Water Treatment SCADA System. Journal of the Korean Institute of Illuminating and Electrical Installation Engineers, 28(2), 24-30.

Kim, S.-H., \& Lee, C.-S. (2010). A Master Data Quality Management Framework. Entrue Journal of Information Technology, 9(2), 109-121. 